\title{
Orbital and suborbital variability in North Atlantic bottom water temperature obtained from deep-sea ostracod $\mathrm{Mg} / \mathrm{Ca}$ ratios
}

\author{
T.M. Cronin ${ }^{\text {a, } *}$, G.S. Dwyer ${ }^{\text {b }}$, P.A. Baker ${ }^{\text {b }}$, J. Rodriguez-Lazaro ${ }^{\text {c }}$, \\ D.M. DeMartino ${ }^{\text {a }}$ \\ a 926 A U.S. Geological Survey, Reston, VA 20192, USA \\ ${ }^{\mathrm{b}}$ Division of Earth and Ocean Sciences, Duke University, Durham, NC 27708, USA \\ ${ }^{\mathrm{c}}$ Department of Paleontology, University Pais Vasco, Bilbao, Spain
}

Received 3 June 1999; accepted for publication 22 March 2000

\begin{abstract}
Magnesium/calcium $(\mathrm{Mg} / \mathrm{Ca})$ ratios were measured in the deep-sea ostracod (Crustacea) genus Krithe from Chain core 82-24-4PC from the western mid-Atlantic Ridge $(3427 \mathrm{~m})$ in order to estimate ocean circulation and bottom water temperature (BWT) variability over the past 200,000 years. $\mathrm{Mg} / \mathrm{Ca}$ ratios have been used as a paleothermometer because the ratios are controlled primarily by ambient water temperatures at the time the organism secretes its adult carapace. Over the past two glacial-interglacial cycles, $\mathrm{Mg} / \mathrm{Ca}$ values oscillated between about $7 \mathrm{mmol} / \mathrm{mol}$ and $12 \mathrm{mmol} / \mathrm{mol}$, equivalent to a BWT range of 0 to $>3.5^{\circ} \mathrm{C}$. The lowest values were obtained on specimens from glacial marine isotope stages (MISs) 2, 4 and 6; the highest values were obtained from specimens from the early part of the Holocene interglacial (MIS 1), and also from MISs 5 and 7. These trends suggest that BWTs in the North Atlantic Ocean fluctuate over orbital time scales.

Suborbital variability in $\mathrm{Mg} / \mathrm{Ca}$ ratios and BWT was also observed for the past 100,000 years. Ratios rose from $\sim 8 \mathrm{mmol} / \mathrm{mol}$ to $\sim 10 \mathrm{mmol} / \mathrm{mol}$ (implying a BWT increase of $\sim 1$ to $3^{\circ} \mathrm{C}$ ) during $14 \mathrm{Mg} / \mathrm{Ca}$ excursions. The highest ratios were found in Krithe dated at approximately 32, 36-38, 43, 48, 73, 85 and $93 \mathrm{ka}$. Although the age model for the Chain 82-24-4PC and temporal resolution do not allow precise correlation, some of these deep-sea bottom temperature excursions appear to correspond to Heinrich events recorded in other regions of the North Atlantic and perhaps Dansgaard-Oeschger interstadial events recorded in Greenland ice cores. If confirmed, this would support the hypothesis that millennial-scale oscillations of climate in the North Atlantic are capable of affecting global climate via thermohaline circulation changes. (C) 2000 Elsevier Science B.V. All rights reserved.
\end{abstract}

Keywords: deep-ocean circulation; magnesium/calcium ratios; North Atlantic Ocean; ostracod; paleoclimate; Quaternary climates

\section{Introduction}

Late Quaternary episodes of abrupt millennialscale climate change were first documented as 'Dansgaard/Oeschger' (D/O) events in Greenland

\footnotetext{
* Corresponding author. Tel.: +1-703-648-6363.

E-mail address: tcronin@usgs.gov (T.M. Cronin)
}

ice core isotopic records (Johnsen et al., 1992; GRIP, 1993; Taylor et al., 1993) and as 'Heinrich events' in oceanic records (Bond et al., 1992; Bond and Lotti, 1995). Millennial-scale climate events are now known from terrestrial records in western (Benson et al., 1996) and southeastern (Grimm et al., 1993) North America, the eastern Pacific Ocean (Behl and Kennett, 1996), the deep North 
Atlantic Ocean (Keigwin and Jones, 1994; Oppo and Lehman, 1995), the ice core record of the Taylor Dome, Antarctica (Steig et al. 1998), as well as older Quaternary records (Raymo et al., 1998), and the Holocene interglacial (Mayewski et al., 1996; Bond et al., 1997). Changes in oceanic thermohaline circulation have been most often proposed as the mechanism causing millennialscale climate change (Broecker, 1994), but other processes such as variations in atmospheric water vapor content and solar insolation may also be involved.

If reorganizations of ocean thermohaline circulation either cause or respond to millennial-scale events, then these should be most directly manifested in the deep North Atlantic Ocean bottom water temperatures (BWTs), which are today influenced by the relative source strength of North Atlantic deep water (NADW) and Antarctic bottom water (AABW). In this paper, we reconstructed late Quaternary North Atlantic BWTs for the last $220 \mathrm{kyr}$ using the magnesium/calcium $(\mathrm{Mg} / \mathrm{Ca})$ paleothermometry method of ostracods. This method utilizes the relationship between water temperature and the concentration of magnesium in the ostracod shell, first recognized by Cadot and Kaesler (1977) and confirmed by Corrége (1993), Corrége and De Deckker (1997), and Dwyer et al. (1995) for deep-sea taxa and by others for coastal and lacustrine species [see De Deckker et al. (1999)]. We present evidence of variability in Krithe $\mathrm{Mg} / \mathrm{Ca}$ ratios indicative of BWT changes of $0-3.5^{\circ} \mathrm{C}$ for orbital time scales and $1-3^{\circ} \mathrm{C}$ for suborbital climatic events.

\section{Material and methods}

We analyzed 245 individual ostracod valves from the Chain core 82-24-4PC $\left(41^{\circ} 43^{\prime} \mathrm{N}, 32^{\circ} 51^{\prime}\right.$, $3427 \mathrm{~m}$ water depth, modern BWT $\sim 2.6^{\circ} \mathrm{C}$ ), located in the path of NADW on the western flank of the mid-Atlantic ridge (Fig. 1). Previous studies of foraminiferal stable isotopes and trace elements $(\mathrm{Cd} / \mathrm{Ca}$ ratios) from this same core have shown that, during glacial and interglacial periods, this region was alternatively influenced by greater amounts of colder AABW and warmer NADW
(Boyle and Keigwin, 1985; Raymo et al., 1992). Thus, it is an excellent site to obtain estimates of relative NADW strength during Quaternary climatic oscillations using $\mathrm{Mg} / \mathrm{Ca}$ paleothermometry. The sediment accumulation rate at the site averages $\sim 3.5 \mathrm{~cm} / \mathrm{kyr}$, so each 2 to $3 \mathrm{~cm}$ thick sample represents roughly 1000 years. We used the age model developed by Boyle and Keigwin (1985) on the basis of oxygen isotope stratigraphy to date the core.

Ostracods are benthic, bivalved Crustacea that secrete a low magnesium calcite shell during moulting. The amount of co-precipitated magnesium in calcite shells of the ubiquitous deep-sea genus Krithe (Coles et al., 1994; Cronin et al., 1999) is largely controlled by the water temperature in which the organism secreted its adult carapace. A positive correlation between $\mathrm{Mg} / \mathrm{Ca}$ and water temperature in Krithe has been observed in independent studies from several different oceans (Cadot and Kaesler, 1977; Corrége, 1993; Corrége and De Deckker, 1997; Dwyer et al., 1995; Cronin et al., 1996; Muller and Nürnberg, 1997). Here we use the $\mathrm{Mg} / \mathrm{Ca}$-temperature equation

BWT $\left({ }^{\circ} \mathrm{C}\right)=(0.854 \times \mathrm{Mg} / \mathrm{Ca})-5.75$

from Dwyer et al. (1995). This equation is based on calibration material collected from between 2 and $14^{\circ} \mathrm{C}$, a range within which $\mathrm{Mg} / \mathrm{Ca}$ ratios vary from about 8 to $22-26 \mathrm{mmol} / \mathrm{mol}$ and has $95 \%$ confidence intervals of $\pm 1.3^{\circ} \mathrm{C}$ for a single downcore specimen. When more than one specimen per sample is analyzed, these limits are reduced by the factor $n^{-1 / 2}$, with $n$ being the number of specimens analyzed. The validity of this calibration has been further supported by new measurements on coretop (Dwyer et al., 2000) and laboratory-cultured specimens (Majoran et al., 2000) of Krithe. Dwyer et al. (2000) also estimated that post mortem dissolution of the ostracod shell can account for only $10-15 \%$ of the variability in $\mathrm{Mg} / \mathrm{Ca}$ ratios in Krithe and that the primary control is temperature as proposed by Cadot and Kaesler (1977), Corrége (1993), Dwyer et al. (1995), and Corrége and De Deckker (1997).

Because $\mathrm{Mg} / \mathrm{Ca}$ ratios in ostracod shells change ontogenetically (Cadot and Kaesler, 1977), we analyzed only adult valves of Krithe. Specimens 


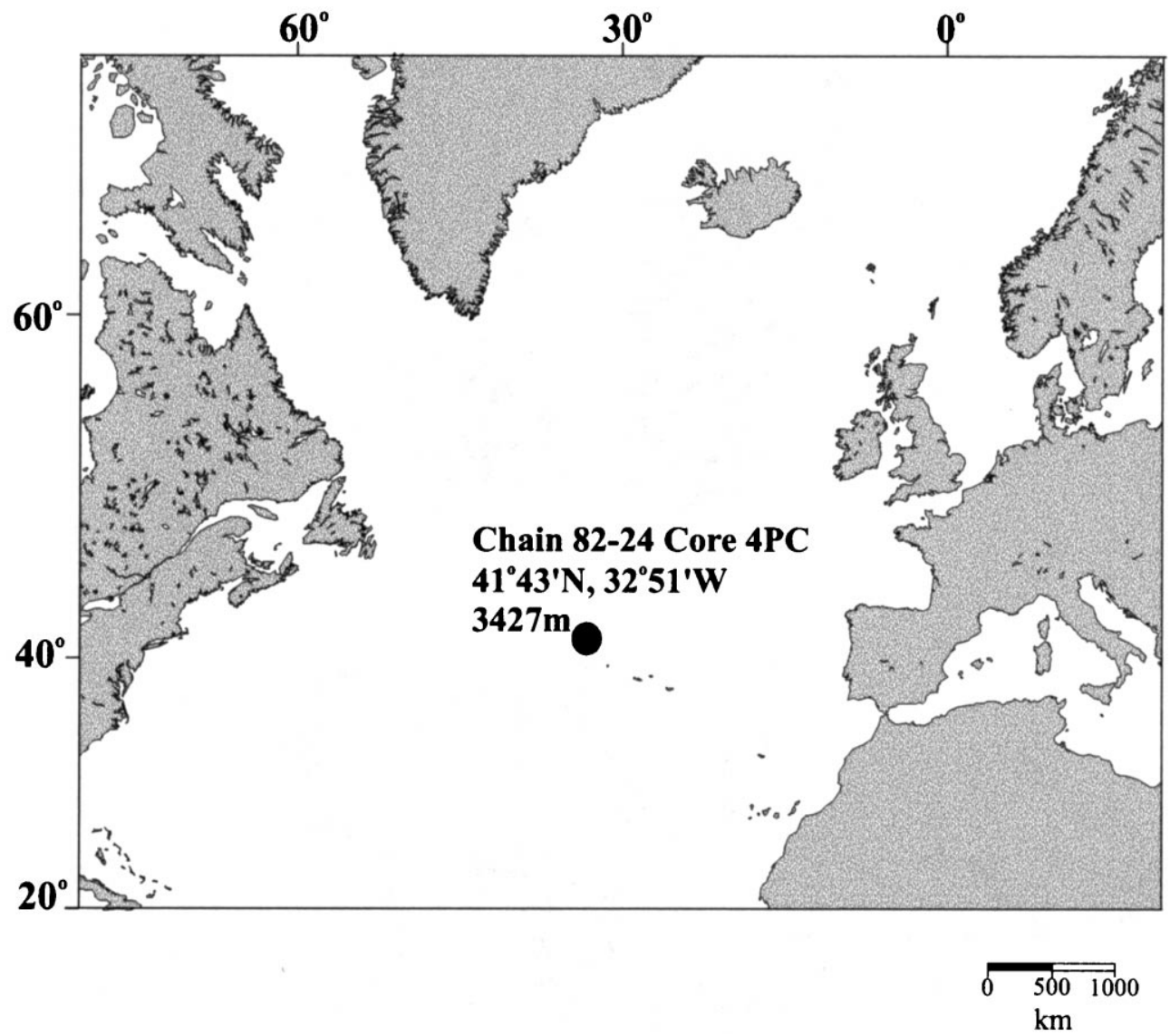

Fig. 1. Map showing location of Chain 82-24-4PC core site on the western side of the mid-Atlantic Ridge, North Atlantic Ocean $\left(41^{\circ} 43^{\prime} \mathrm{N}, 32^{\circ} 51^{\prime} \mathrm{W}\right)$.

from Chain 82-24 sediment samples, provided by E. Boyle and L. Keigwin, were soaked in clorox overnight to remove organic material and other debris. They were then washed and sonicated in a two-step bath of deionized water, before dissolving in 4 to $30 \mathrm{ml}$ of $0.05 \mathrm{~N}$ nitric acid, depending on the weight of each valve. One to three individuals per sample were analyzed on the Duke University Spectrascan 7 direct current plasma (DCP) emission spectrometer for magnesium and calcium. The analytical precision was $\pm 3 \%$ based on replicate analyses of samples and standards. An in-house limestone standard Duke PE3 yielded $\mathrm{Mg} / \mathrm{Ca}$ of $8.50 \pm 0.04 \mathrm{mmol} / \mathrm{mol}(1 \sigma)(n=40)$. The measured $\mathrm{Mg} / \mathrm{Ca}$ ratios for the Krithe valves and estimated BWTs are given in Table 1.

\section{Results}

\subsection{Orbital-scale glacial-interglacial variability}

The results show that large amplitude oscillations in the $\mathrm{Mg} / \mathrm{Ca}$ of ostracod shells and estimated BWT in the North Atlantic occur at orbital time scales (Fig. 2). Glacial-age $\mathrm{Mg} / \mathrm{Ca}$ ratios and estimated BWTs for marine isotope stages 6,4 and 2 were respectively $\sim 6.5$ to $7.0 \mathrm{mmol} / \mathrm{mol}$ and $0^{\circ} \mathrm{C}$. Moreover, $\mathrm{Mg} / \mathrm{Ca}$ variability generally coincides with changes in benthic foraminiferal values of $\delta^{18} \mathrm{O}, \delta^{13} \mathrm{C}$, and $\mathrm{Cd} / \mathrm{Ca}$ measured on subsamples from the same interval by Boyle and Keigwin (1985). Low $\mathrm{Mg} / \mathrm{Ca}$ ratios coincide with relatively high foraminiferal $\mathrm{Cd} / \mathrm{Ca}$ ratios and low $\delta^{13} \mathrm{C}$ 
Table 1

$\mathrm{Mg} / \mathrm{Ca}$ ratios in Krithe from the Chain $82-24-4 \mathrm{PC}$ core

\begin{tabular}{|c|c|c|c|c|c|c|c|}
\hline $\begin{array}{l}\text { Depth interval } \\
(\mathrm{cm})^{\mathrm{a}}\end{array}$ & $\begin{array}{l}\text { Core depth midpoint } \\
(\mathrm{cm})^{\mathrm{b}}\end{array}$ & $\begin{array}{l}\text { Age } \\
(\mathrm{ka})\end{array}$ & Sex & $\mathrm{VPI}^{\mathrm{c}}$ & $\begin{array}{l}\mathrm{Mg} / \mathrm{Ca} \\
(\mathrm{mmol} / \mathrm{mol})\end{array}$ & $\begin{array}{l}\text { Mean } \\
\mathrm{Mg} / \mathrm{Ca}^{\mathrm{d}}\end{array}$ & $\begin{array}{l}\text { Estimated } \\
\text { BWT }^{\mathrm{e}}\end{array}$ \\
\hline $1-4$ & 2.5 & 0.69 & $\mathrm{~F}$ & 3 & 9.84 & 9.84 & 2.66 \\
\hline $4-7$ & 5.5 & 1.51 & $\mathrm{M}$ & 3 & 10.20 & 10.24 & 3.00 \\
\hline $4-7$ & 5.5 & 1.51 & $\mathrm{~F}$ & 4 & 10.29 & & \\
\hline $7-10$ & 8.5 & 2.34 & $\mathrm{~F}$ & 5 & 9.87 & 10.85 & 3.52 \\
\hline $7-10$ & 8.5 & 2.34 & $\mathrm{~F}$ & 4 & 11.84 & & \\
\hline $10-13$ & 11.5 & 3.16 & $\mathrm{~F}$ & 4 & 10.21 & 11.02 & 3.66 \\
\hline $10-13$ & 11.5 & 3.16 & $\mathrm{~F}$ & 5 & 11.82 & & \\
\hline $13-16$ & 14.5 & 3.99 & $\mathrm{~F}$ & 5 & 8.97 & 9.99 & 2.79 \\
\hline $13-16$ & 14.5 & 3.99 & $\mathrm{M}$ & 4 & 11.01 & & \\
\hline $16-19$ & 17.5 & 4.81 & $\mathrm{~F}$ & 2 & 9.52 & 9.52 & 2.38 \\
\hline $19-22$ & 20.5 & 5.64 & $\mathrm{~F}$ & 3 & 9.27 & 9.84 & 2.65 \\
\hline $20-21$ & 20.5 & 5.64 & $\mathrm{~F}$ & 1 & 10.67 & & \\
\hline $20-21$ & 20.5 & 5.64 & $\mathrm{~F}$ & 4 & 9.57 & & \\
\hline $22-25$ & 23.5 & 6.46 & $\mathrm{~F}$ & 1 & 10.95 & 10.95 & 3.61 \\
\hline $25-27$ & 26 & 7.15 & $\mathrm{M}$ & 1 & 12.54 & 12.54 & 4.96 \\
\hline $29-33$ & 31 & 8.53 & $\mathrm{~F}$ & 1 & 11.51 & 12.05 & 4.54 \\
\hline $29-33$ & 31 & 8.53 & $\mathrm{M}$ & 2 & 12.59 & & \\
\hline $31-32$ & 31.5 & 8.66 & $\mathrm{~F}$ & 1 & 9.95 & 9.95 & 2.75 \\
\hline $34-37$ & 35.5 & 9.76 & $\mathrm{M}$ & 1 & 12.67 & 12.65 & 5.06 \\
\hline $34-37$ & 35.5 & 9.76 & $\mathrm{~F}$ & 1 & 12.63 & & \\
\hline $40-42$ & 41 & 11.29 & $\mathrm{~F}$ & 2 & 11.32 & 11.32 & 3.92 \\
\hline $47-50$ & 48.5 & 13.48 & M & 1 & 11.33 & 11.33 & 3.93 \\
\hline $50-52.5$ & 51.25 & 14.28 & $\mathrm{~F}$ & 3 & 9.34 & 9.34 & 2.23 \\
\hline $52.5-54.5$ & 53.5 & 14.94 & $\mathrm{~F}$ & 2 & 10.76 & 12.03 & 4.52 \\
\hline $52.5-54.5$ & 53.5 & 14.94 & $\mathrm{M}$ & 3 & 13.29 & & \\
\hline $56-59$ & 57.5 & 16.10 & $\mathrm{~F}$ & 3 & 10.19 & 10.19 & 2.96 \\
\hline $57.5-58.5$ & 58 & 16.25 & $\mathrm{M}$ & 2 & 9.22 & 9.22 & 2.12 \\
\hline $59-62$ & 60.5 & 16.98 & $\mathrm{~F}$ & 6 & 7.46 & 6.66 & -0.06 \\
\hline $59-62$ & 60.5 & 16.98 & $\mathrm{~F}$ & 4 & 5.86 & & \\
\hline $62-65$ & 63.5 & 17.85 & $\mathrm{~F}$ & 5 & 8.03 & 8.75 & 1.73 \\
\hline $62-65$ & 63.5 & 17.85 & $\mathrm{M}$ & 6 & 8.34 & & \\
\hline $62-65$ & 63.5 & 17.85 & $\mathrm{M}$ & 5 & 8.79 & & \\
\hline $62-65$ & 63.5 & 17.85 & $\mathrm{~F}$ & 4 & 9.07 & & \\
\hline $62-65$ & 63.5 & 17.85 & $\mathrm{~F}$ & 4 & 9.71 & & \\
\hline $62-65$ & 63.5 & 17.85 & $\mathrm{~F}$ & 5 & 8.59 & & \\
\hline $65-68$ & 66.5 & 18.58 & $\mathrm{~F}$ & 4 & 9.00 & 7.75 & 0.87 \\
\hline $65-68$ & 66.5 & 18.58 & $\mathrm{~F}$ & 5 & 6.56 & & \\
\hline $65-68$ & 66.5 & 18.58 & $\mathrm{~F}$ & 7 & 7.68 & & \\
\hline $68-71$ & 69.5 & 19.27 & $\mathrm{~F}$ & 5 & 9.26 & 9.63 & 2.48 \\
\hline $68-71$ & 69.5 & 19.27 & $\mathrm{~F}$ & 6 & 9.27 & & \\
\hline $68-71$ & 69.5 & 19.27 & $\mathrm{~F}$ & 6 & 10.36 & & \\
\hline $75-78$ & 76.5 & 20.88 & & 7 & 6.47 & 6.47 & -0.22 \\
\hline $80-83$ & 81.5 & 22.04 & $\mathrm{~F}$ & 6 & 7.96 & 8.25 & 1.30 \\
\hline $80-83$ & 81.5 & 22.04 & $\mathrm{~F}$ & 6 & 8.55 & & \\
\hline $83-85$ & 84 & 22.62 & $?$ & 6 & 9.96 & 9.96 & 2.76 \\
\hline $85-87$ & 86 & 23.08 & $\mathrm{M}$ & 3 & 9.49 & 9.36 & 2.25 \\
\hline $85-87$ & 86 & 23.08 & $\mathrm{~F}$ & 4 & 9.24 & & \\
\hline $88-91$ & 89.5 & 23.88 & $\mathrm{M}$ & 6 & 9.47 & 9.24 & 2.14 \\
\hline $88-91$ & 89.5 & 23.88 & $\mathrm{~F}$ & 6 & 8.32 & & \\
\hline $88-91$ & 89.5 & 23.88 & $\mathrm{M}$ & 5 & 9.93 & & \\
\hline $91-94$ & 92.5 & 24.72 & $\mathrm{~F}$ & DND & 8.76 & 8.76 & 1.74 \\
\hline $94-97$ & 95.5 & 25.58 & $\mathrm{~F}$ & DND & 7.60 & 7.60 & 0.74 \\
\hline
\end{tabular}


Table 1 (continued).

\begin{tabular}{|c|c|c|c|c|c|c|c|}
\hline $\begin{array}{l}\text { Depth interval } \\
(\mathrm{cm})^{\mathrm{a}}\end{array}$ & $\begin{array}{l}\text { Core depth midpoint } \\
(\mathrm{cm})^{\mathrm{b}}\end{array}$ & $\begin{array}{l}\text { Age } \\
(\mathrm{ka})\end{array}$ & Sex & $\mathrm{VPI}^{\mathrm{c}}$ & $\begin{array}{l}\mathrm{Mg} / \mathrm{Ca} \\
(\mathrm{mmol} / \mathrm{mol})\end{array}$ & $\begin{array}{l}\text { Mean } \\
\mathrm{Mg} / \mathrm{Ca}^{\mathrm{d}}\end{array}$ & $\begin{array}{l}\text { Estimated } \\
\text { BWT }^{\mathrm{e}}\end{array}$ \\
\hline $99-100$ & 99.5 & 26.73 & $\mathrm{~F}$ & 5 & 9.08 & 9.08 & 2.01 \\
\hline $100-103$ & 101.5 & 27.30 & $\mathrm{~F}$ & DND & 9.13 & 9.13 & 2.05 \\
\hline $104-107$ & 105.5 & 28.45 & M & DND & 7.89 & 7.89 & 0.99 \\
\hline $107-110$ & 108.5 & 29.31 & DND & 9.00 & 9.00 & 1.94 & \\
\hline $112-114$ & 113 & 30.60 & $\mathrm{~F}$ & DND & 8.30 & 8.30 & 1.34 \\
\hline $116.5-119$ & 117.75 & 31.96 & $\mathrm{M}$ & DND & 7.86 & 7.86 & 0.97 \\
\hline $120-121$ & 120.5 & 32.75 & $\mathrm{~F}$ & 6 & 9.10 & 9.05 & 1.98 \\
\hline $119-122$ & 120.5 & 32.75 & $\mathrm{~F}$ & DND & 9.01 & & \\
\hline $123-126$ & 124.5 & 33.90 & $\mathrm{~F}$ & DND & 7.51 & 7.51 & 0.66 \\
\hline $126-130$ & 128 & 34.90 & $\mathrm{M}$ & DND & 8.68 & 8.68 & 1.66 \\
\hline $130-133$ & 131.5 & 35.91 & M & DND & 12.00 & 12.00 & 4.50 \\
\hline $138.5-141$ & 139.75 & 38.27 & DND & 8.57 & 8.57 & 1.57 & \\
\hline $140-142$ & 141 & 38.63 & $\mathrm{M}$ & 5 & 9.61 & 9.61 & 2.46 \\
\hline $141-143.5$ & 142.25 & 38.99 & DND & 8.93 & 8.93 & 1.88 & \\
\hline $143.5-146$ & 144.75 & 39.71 & $\mathrm{~F}$ & 3 & 8.61 & 8.61 & 1.60 \\
\hline $146-148$ & 147 & 40.35 & $\mathrm{~F}$ & 2 & 8.99 & 8.99 & 1.93 \\
\hline $148-150.5$ & 149.25 & 41.00 & $\mathrm{~F}$ & 3 & 6.85 & 6.85 & 0.10 \\
\hline $150.5-153$ & 151.75 & 41.72 & M & 4 & 9.58 & 9.58 & 2.43 \\
\hline $153-155$ & 154 & 42.36 & $\mathrm{~F}$ & 4 & 10.09 & 10.09 & 2.87 \\
\hline $155-157$ & 156.25 & 43.01 & $\mathrm{~F}$ & 5 & 8.28 & 8.28 & 1.32 \\
\hline $157.5-159.5$ & 158.5 & 43.65 & M & 3 & 11.73 & 11.73 & 4.27 \\
\hline $159.5-162$ & 160.75 & 44.30 & $\mathrm{~F}$ & 4 & 9.69 & 9.69 & 2.53 \\
\hline $161-162$ & 161.5 & 44.51 & $\mathrm{~F}$ & 5 & 8.71 & 8.71 & 1.69 \\
\hline $162-164.5$ & 163.25 & 45.02 & $\mathrm{~F}$ & 4 & 8.18 & 8.18 & 1.24 \\
\hline $164.5-167$ & 165.75 & 45.73 & $\mathrm{~F}$ & 4 & 10.41 & 8.95 & 1.89 \\
\hline $164.5-167$ & 165.75 & 45.73 & $\mathrm{~F}$ & 3 & 7.48 & & \\
\hline $175-177$ & 176 & 48.67 & M & 2 & 10.56 & 10.56 & 3.27 \\
\hline $177-179.5$ & 178.25 & 49.32 & $\mathrm{~F}$ & 2 & 8.07 & 8.07 & 1.15 \\
\hline $178-180$ & 179 & 49.53 & & 2 & 8.75 & 9.64 & 2.48 \\
\hline $178-180$ & 179 & 49.53 & 1 & 10.00 & & & \\
\hline $178-180$ & 179 & 49.53 & 1 & 10.17 & & & \\
\hline $179.5-181.5$ & 180.5 & 49.96 & M & 2 & 9.40 & 9.40 & 2.28 \\
\hline $181.5-184.5$ & 183 & 50.68 & $\mathrm{~F}$ & 2 & 9.16 & 9.16 & 2.07 \\
\hline $184.5-187$ & 185.75 & 51.47 & $\mathrm{~F}$ & 2 & 9.80 & 9.52 & 2.39 \\
\hline $184.5-187$ & 185.75 & 51.47 & $\mathrm{~F}$ & 2 & 9.25 & & \\
\hline $187-190$ & 188.5 & 52.26 & M & 3 & 10.73 & 10.73 & 3.42 \\
\hline $190-193$ & 191.5 & 53.12 & M & 2 & 10.65 & 9.05 & 1.98 \\
\hline $190-193$ & 191.5 & 53.12 & $\mathrm{~F}$ & 2 & 7.44 & & \\
\hline $193-195$ & 194 & 53.84 & M & 4 & 9.58 & 9.58 & 2.43 \\
\hline $195-198$ & 197 & 54.70 & M & 4 & 9.59 & 8.87 & 1.83 \\
\hline $195-198$ & 197 & 54.70 & $\mathrm{~F}$ & 3 & 8.16 & & \\
\hline $198-201$ & 199.5 & 55.41 & $\mathrm{~F}$ & 5 & 9.65 & 9.82 & 2.64 \\
\hline $198-201$ & 199.5 & 55.41 & M & 5 & 10.00 & & \\
\hline $201-203$ & 202 & 56.13 & $\mathrm{~F}$ & 3 & 9.10 & 9.10 & 2.03 \\
\hline $203-205$ & 204 & 56.71 & M & 3 & 10.56 & 10.56 & 3.27 \\
\hline $207-209$ & 208 & 57.85 & $\mathrm{~F}$ & 3 & 8.99 & 8.99 & 1.93 \\
\hline $218-220$ & 219 & 61.72 & M & 2 & 9.14 & 9.14 & 2.06 \\
\hline $230-233$ & 231.5 & 68.87 & $\mathrm{~F}$ & 4 & 5.53 & 5.53 & -1.02 \\
\hline $233-236$ & 234.5 & 68.62 & M & 4 & 7.45 & 7.45 & 0.62 \\
\hline $236-239.5$ & 237.75 & 70.52 & $\mathrm{M}$ & 5 & 9.36 & 8.99 & 1.93 \\
\hline $236-239.5$ & 237.75 & 70.52 & M & 6 & 8.62 & & \\
\hline $239.5-241.5$ & 240.5 & 72.12 & $\mathrm{~F}$ & 4 & 7.30 & 8.16 & 1.22 \\
\hline $239.5-241.5$ & 240.5 & 72.12 & $\mathrm{~F}$ & 4 & 9.02 & & \\
\hline
\end{tabular}


Table 1 (continued).

\begin{tabular}{|c|c|c|c|c|c|c|c|}
\hline $\begin{array}{l}\text { Depth interval } \\
(\mathrm{cm})^{\mathrm{a}}\end{array}$ & $\begin{array}{l}\text { Core depth midpoint } \\
(\mathrm{cm})^{\mathrm{b}}\end{array}$ & $\begin{array}{l}\text { Age } \\
\text { (ka) }\end{array}$ & Sex & $\mathrm{VPI}^{\mathrm{c}}$ & $\begin{array}{l}\mathrm{Mg} / \mathrm{Ca} \\
(\mathrm{mmol} / \mathrm{mol})\end{array}$ & $\begin{array}{l}\text { Mean } \\
\mathrm{Mg} / \mathrm{Ca}^{\mathrm{d}}\end{array}$ & $\begin{array}{l}\text { Estimated } \\
\text { BWT }^{\mathrm{e}}\end{array}$ \\
\hline $241.5-243$ & 242.25 & 73.06 & $\mathrm{~F}$ & 4 & 8.07 & 8.07 & 1.14 \\
\hline $242-243$ & 243 & 73.26 & $\mathrm{~F}$ & 5 & 8.52 & 8.28 & 1.32 \\
\hline $242-243$ & 243 & 73.26 & $\mathrm{~F}$ & 6 & 8.04 & & \\
\hline $244-246$ & 245 & 73.77 & $\mathrm{~F}$ & 4 & 8.27 & 8.27 & 1.32 \\
\hline $246-248$ & 247 & 74.28 & $\mathrm{~F}$ & 5 & 9.37 & 9.37 & 2.25 \\
\hline $248-250$ & 249 & 74.79 & $\mathrm{M}$ & 4 & 9.65 & 9.65 & 2.49 \\
\hline $252-254$ & 253 & 75.82 & $\mathrm{~F}$ & 5 & 8.81 & 8.81 & 1.78 \\
\hline $254-256$ & 255 & 76.33 & $\mathrm{~F}$ & 5 & 9.98 & 9.98 & 2.77 \\
\hline $256-258$ & 257 & 76.84 & $\mathrm{~F}$ & 5 & 8.87 & 8.87 & 1.82 \\
\hline $258-259$ & 258.5 & 77.23 & $\mathrm{~F}$ & 4 & 10.07 & 9.22 & 2.12 \\
\hline $258-259$ & 258.5 & 77.23 & $\mathrm{M}$ & 4 & 8.36 & & \\
\hline $258-260$ & 259 & 77.35 & $\mathrm{M}$ & 4 & 8.18 & 8.18 & 1.24 \\
\hline $260-263$ & 261.5 & 77.99 & $\mathrm{~F}$ & 4 & 9.45 & 9.45 & 2.32 \\
\hline $263-265$ & 264 & 78.63 & $\mathrm{~F}$ & 3 & 9.40 & 9.40 & 2.28 \\
\hline $267.5-271$ & 269.25 & 79.98 & $\mathrm{~F}$ & 3 & 8.13 & 8.13 & 1.20 \\
\hline $271-275$ & 273 & 80.94 & $\mathrm{~F}$ & 4 & 9.83 & 9.83 & 2.64 \\
\hline $275-279$ & 277 & 81.96 & $\mathrm{~F}$ & 3 & 8.19 & 8.19 & 1.25 \\
\hline $280-281$ & 280.5 & 82.86 & $\mathrm{M}$ & 2 & 11.31 & 11.31 & 3.91 \\
\hline $281-283$ & 282 & 83.24 & $\mathrm{~F}$ & 3 & 9.94 & 9.94 & 2.74 \\
\hline $283-284.5$ & 283.75 & 83.69 & $\mathrm{~F}$ & 3 & 8.37 & 8.37 & 1.40 \\
\hline $284.5-286$ & 285.25 & 84.08 & M & 2 & 10.57 & 10.57 & 3.28 \\
\hline $288-290$ & 289 & 85.04 & $\mathrm{~F}$ & 1 & 9.59 & 9.59 & 2.44 \\
\hline $290-292$ & 291 & 85.55 & M & 2 & 11.00 & 11.00 & 3.64 \\
\hline $292-294$ & 293 & 86.06 & $\mathrm{~F}$ & 3 & 10.07 & 10.07 & 2.85 \\
\hline $295.5-298.5$ & 297 & 87.09 & $\mathrm{~F}$ & 4 & 8.51 & 8.51 & 1.52 \\
\hline $301-304$ & 303.5 & 88.75 & $\mathrm{~F}$ & 4 & 8.82 & 8.82 & 1.79 \\
\hline $304-307$ & 305.5 & 89.26 & $\mathrm{M}$ & 3 & 10.08 & 10.08 & 2.86 \\
\hline $307-311$ & 309 & 90.16 & $\mathrm{~F}$ & 3 & 8.67 & 8.67 & 1.66 \\
\hline $311-313.5$ & 312.25 & 90.99 & $\mathrm{~F}$ & 4 & 7.48 & 7.48 & 0.64 \\
\hline $313.5-316$ & 314.75 & 91.63 & $\mathrm{M}$ & 3 & 9.39 & 9.39 & 2.27 \\
\hline $316-319$ & 317.5 & 92.34 & $\mathrm{~F}$ & 4 & 8.65 & 8.65 & 1.64 \\
\hline $320-321$ & 320.5 & 93.10 & $\mathrm{~F}$ & 4 & 9.97 & 9.21 & 2.12 \\
\hline $319-322$ & 320.5 & 93.10 & $\mathrm{~F}$ & lost & 8.46 & & \\
\hline $322-323.75$ & 323.75 & 93.94 & $\mathrm{~F}$ & 4 & 7.03 & 7.03 & 0.26 \\
\hline $325.5-328$ & 326.75 & 94.70 & $\mathrm{~F}$ & 3 & 7.67 & 7.65 & 0.79 \\
\hline $325.5-328$ & 326.75 & 94.70 & $\mathrm{~F}$ & 4 & 7.63 & & \\
\hline $328-331$ & 329.5 & 95.41 & M & 4 & 8.15 & 8.09 & 1.16 \\
\hline $328-331$ & 329.5 & 95.41 & $\mathrm{~F}$ & 4 & 8.02 & & \\
\hline $331-334$ & 332.5 & 96.18 & $\mathrm{M}$ & 5 & 8.20 & 8.20 & 1.25 \\
\hline $334-337$ & 335.5 & 96.95 & $\mathrm{~F}$ & 3 & 8.38 & 8.38 & 1.41 \\
\hline $337-340$ & 338.5 & 97.71 & $\mathrm{M}$ & 1 & 8.41 & 8.41 & 1.43 \\
\hline $340-342$ & 341 & 98.35 & $\mathrm{~F}$ & 3 & 10.67 & 10.67 & 3.36 \\
\hline $340-343$ & 341.5 & 98.48 & $\mathrm{~F}$ & 3 & 8.81 & 8.81 & 1.78 \\
\hline $343-345$ & 344 & 99.12 & $\mathrm{M}$ & 2 & 11.17 & 11.17 & 3.79 \\
\hline $345-348$ & 346.5 & 99.76 & $\mathrm{~F}$ & 2 & 10.04 & 10.04 & 2.82 \\
\hline $380-383.5$ & 349.75 & 100.59 & $\mathrm{FF}$ & $3 / 3$ & 8.93 & 8.62 & 1.61 \\
\hline $380-383.5$ & 349.75 & 100.59 & $\mathrm{FF}$ & $3 / 3$ & 8.31 & & \\
\hline $383-384$ & 351.5 & 101.04 & $\mathrm{~F}$ & 1 & 11.45 & 11.45 & 4.03 \\
\hline $383.5-386.5$ & 353 & 101.43 & $\mathrm{~F}$ & 4 & 10.24 & 10.24 & 3.00 \\
\hline $386.5-390$ & 356.25 & 102.26 & $\mathrm{M}$ & 3 & 8.40 & 8.40 & 1.43 \\
\hline $391-394.5$ & 360.75 & 103.41 & $\mathrm{~F}$ & 2 & 8.84 & 8.84 & 1.80 \\
\hline $396-398$ & 365 & 104.50 & $\mathrm{~F}$ & 3 & 8.46 & 8.46 & 1.48 \\
\hline $398-400$ & 367 & 105.01 & $\mathrm{~F}$ & 4 & 7.74 & 7.74 & 0.87 \\
\hline
\end{tabular}


Table 1 (continued).

\begin{tabular}{|c|c|c|c|c|c|c|c|}
\hline $\begin{array}{l}\text { Depth interval } \\
(\mathrm{cm})^{\mathrm{a}}\end{array}$ & $\begin{array}{l}\text { Core depth midpoint } \\
(\mathrm{cm})^{\mathrm{b}}\end{array}$ & $\begin{array}{l}\text { Age } \\
\text { (ka) }\end{array}$ & Sex & $\mathrm{VPI}^{\mathrm{c}}$ & $\begin{array}{l}\mathrm{Mg} / \mathrm{Ca} \\
(\mathrm{mmol} / \mathrm{mol})\end{array}$ & $\begin{array}{l}\text { Mean } \\
\mathrm{Mg} / \mathrm{Ca}^{\mathrm{d}}\end{array}$ & $\begin{array}{l}\text { Estimated } \\
\text { BWT }^{\mathrm{e}}\end{array}$ \\
\hline $400-402$ & 369 & 105.52 & $\mathrm{~F}$ & 3 & 9.12 & 9.12 & 2.05 \\
\hline $402-404$ & 371 & 106.04 & $\mathrm{~F}$ & 2 & 9.51 & 9.51 & 2.38 \\
\hline $404.5-407$ & 373.75 & 106.74 & $\mathrm{M}$ & 2 & 11.89 & 11.89 & 4.40 \\
\hline $408-409$ & 376.5 & 107.45 & $\mathrm{~F}$ & frag & 9.88 & 9.88 & 2.69 \\
\hline $410-413$ & 379.5 & 108.21 & $\mathrm{~F}$ & lost & 9.50 & 9.50 & 2.37 \\
\hline $413-415.5$ & 382.25 & 108.92 & $\mathrm{~F}$ & 3 & 8.70 & 8.70 & 1.69 \\
\hline $415.5-418$ & 384.75 & 109.56 & $\mathrm{~F}$ & DND & 8.96 & 8.99 & 1.93 \\
\hline $415.5-418$ & 384.75 & 109.56 & $\mathrm{~F}$ & DND & 9.03 & & \\
\hline $418-420.5$ & 387.25 & 110.20 & $\mathrm{M}$ & 4 & 8.02 & 8.02 & 1.11 \\
\hline $421-424$ & 390.5 & 111.03 & $\mathrm{~F}$ & 4 & 8.91 & 8.91 & 1.86 \\
\hline $423.5-426$ & 392.75 & 111.61 & $\mathrm{~F}$ & 3 & 9.07 & 9.15 & 2.06 \\
\hline $423.5-426$ & 392.75 & 111.61 & $\mathrm{~F}$ & 4 & 9.22 & & \\
\hline $422-428$ & 393 & 111.67 & M & 4 & 7.74 & 8.38 & 1.41 \\
\hline $422-428$ & 393 & 111.67 & $\mathrm{~F}$ & 5 & 9.01 & & \\
\hline $426-429$ & 395.5 & 112.31 & $\mathrm{~F}$ & 4 & 7.36 & 8.48 & 1.49 \\
\hline $426-429$ & 395.5 & 112.31 & M & 5 & 9.60 & & \\
\hline $429-432$ & 398.5 & 113.08 & & DND & 8.86 & 9.08 & 2.01 \\
\hline $429-432$ & 398.5 & 113.08 & $\mathrm{~F}$ & 4 & 9.31 & & \\
\hline $432-435$ & 401.5 & 113.85 & M & DND & 8.16 & 8.59 & 1.59 \\
\hline $432-435$ & 401.5 & 113.85 & $\mathrm{~F}$ & 5 & 9.19 & & \\
\hline $432-435$ & 401.5 & 113.85 & M & 5 & 8.41 & & \\
\hline $435-438$ & 404.5 & 114.62 & M & 5 & 10.31 & 9.00 & 1.94 \\
\hline $435-438$ & 404.5 & 114.62 & M & 5 & 7.69 & & \\
\hline $438-440$ & 407 & 115.32 & $\mathrm{~F}$ & 6 & 7.41 & 8.40 & 1.42 \\
\hline $438-440$ & 407 & 115.32 & $\mathrm{~F}$ & 4 & 9.39 & & \\
\hline $440-441$ & 408.5 & 115.81 & $\mathrm{~F}$ & 5 & 9.47 & 8.33 & 1.37 \\
\hline $440-441$ & 408.5 & 115.81 & $\mathrm{~F}$ & 5 & 7.19 & & \\
\hline $440-443$ & 409.5 & 116.13 & $\mathrm{M}$ & 4 & 10.76 & 10.76 & 3.44 \\
\hline $443-446$ & 412.5 & 117.11 & & DND & 8.45 & 8.45 & 1.47 \\
\hline $443-446$ & 412.5 & 117.11 & $\mathrm{M}$ & 5 & 8.67 & & \\
\hline $443-446$ & 412.5 & 117.11 & $\mathrm{~F}$ & 4 & 8.24 & & \\
\hline $446-449$ & 415.5 & 118.08 & $\mathrm{~F}$ & DND & 10.77 & 9.54 & 2.40 \\
\hline $446-449$ & 415.5 & 118.08 & M & 4 & 8.88 & & \\
\hline $446-449$ & 415.5 & 118.08 & $\mathrm{M}$ & 4 & 8.97 & & \\
\hline $453-456$ & 422.5 & 120.35 & $\mathrm{~F}$ & DND & 10.32 & 10.24 & 3.00 \\
\hline $453-456$ & 422.5 & 120.35 & M & 4 & 9.51 & & \\
\hline $453-456$ & 422.5 & 120.35 & $\mathrm{M}$ & 5 & 10.89 & & \\
\hline $456-459$ & 425.5 & 121.32 & $\mathrm{~F}$ & DND & 10.57 & 11.10 & 3.73 \\
\hline $456-459$ & 425.5 & 121.32 & $\mathrm{~F}$ & 4 & 11.37 & & \\
\hline $456-459$ & 425.5 & 121.32 & $\mathrm{M}$ & 3 & 11.36 & & \\
\hline $459-461$ & 428 & 122.13 & $\mathrm{~F}$ & DND & 9.79 & 10.39 & 3.13 \\
\hline $459-461$ & 428 & 122.13 & $\mathrm{M}$ & 4 & 10.97 & & \\
\hline $459-461$ & 428 & 122.13 & $\mathrm{M}$ & 4 & 10.41 & & \\
\hline $460-461$ & 428.5 & 122.30 & $\mathrm{M}$ & 3 & 10.54 & 10.25 & 3.00 \\
\hline $460-461$ & 428.5 & 122.30 & $\mathrm{~F}$ ? & 4 & 9.95 & & \\
\hline $461-464$ & 430.5 & 122.94 & $\mathrm{~F}$ & 3 & 9.59 & 9.59 & 2.45 \\
\hline $462-465$ & 431.5 & 123.27 & M? & 5 & 11.14 & 10.24 & 3.00 \\
\hline $462-465$ & 431.5 & 123.27 & $\mathrm{~F}$ & 4 & 9.34 & & \\
\hline $464-467$ & 433.5 & 123.92 & $\mathrm{~F}$ & 4 & 9.83 & 9.83 & 2.65 \\
\hline $467-470$ & 436.5 & 124.89 & M & DND & 7.66 & 8.47 & 1.49 \\
\hline $467-470$ & 436.5 & 124.89 & $\mathrm{~F}$ & 5 & 10.16 & & \\
\hline $467-470$ & 436.5 & 124.89 & $\mathrm{~F}$ & 3 & 9.91 & & \\
\hline $467-470$ & 436.5 & 124.89 & $\mathrm{~F}$ & 4 & 6.15 & & \\
\hline
\end{tabular}


Table 1 (continued).

\begin{tabular}{|c|c|c|c|c|c|c|c|}
\hline $\begin{array}{l}\text { Depth interval } \\
(\mathrm{cm})^{\mathrm{a}}\end{array}$ & $\begin{array}{l}\text { Core depth midpoint } \\
(\mathrm{cm})^{\mathrm{b}}\end{array}$ & $\begin{array}{l}\text { Age } \\
(\mathrm{ka})\end{array}$ & Sex & $\mathrm{VPI}^{\mathrm{c}}$ & $\begin{array}{l}\mathrm{Mg} / \mathrm{Ca} \\
(\mathrm{mmol} / \mathrm{mol})\end{array}$ & $\begin{array}{l}\text { Mean } \\
\mathrm{Mg} / \mathrm{Ca}^{\mathrm{d}}\end{array}$ & $\begin{array}{l}\text { Estimated } \\
\text { BWT }^{\mathrm{e}}\end{array}$ \\
\hline $470-472.5$ & 439.25 & 125.78 & M & 4.00 & 8.90 & 8.90 & 1.85 \\
\hline $472.5-475.5$ & 442 & 126.67 & $\mathrm{~F}$ & 5.00 & 9.38 & 9.11 & 2.04 \\
\hline $472.5-475.5$ & 442 & 126.67 & $\mathrm{~F}$ & 6.00 & 8.84 & & \\
\hline $476-478.5$ & 445.25 & 127.93 & $\mathrm{~F}$ & 5.00 & 8.64 & 8.64 & 1.63 \\
\hline $478.5-482$ & 448.25 & 129.18 & $\mathrm{~F}$ & 4.00 & 8.55 & 9.16 & 2.07 \\
\hline $478.5-482$ & 448.25 & 129.18 & M & 5.00 & 9.77 & & \\
\hline $480-481$ & 448.5 & 129.28 & M & 4 & 6.76 & 6.76 & 0.03 \\
\hline $482-485$ & 451.5 & 130.53 & $\mathrm{~F}$ & 4.00 & 6.64 & 6.64 & -0.07 \\
\hline $485.5-489$ & 455.25 & 132.09 & $\mathrm{~F}$ & 5.00 & 6.58 & 6.36 & -0.32 \\
\hline $485.5-489$ & 455.25 & 132.09 & $\mathrm{~F}$ & 4.00 & 6.13 & & \\
\hline $489-492$ & 458.5 & 133.44 & $\mathrm{~F}$ & 4.00 & 8.22 & 9.13 & 2.05 \\
\hline $489-492$ & 458.5 & 133.44 & $\mathrm{~F}$ & 5.00 & 10.05 & & \\
\hline $489-493$ & 459 & 133.65 & & DND & 6.67 & 6.67 & -0.05 \\
\hline $493-496$ & 462.5 & 135.10 & $\mathrm{~F}$ & DND & 8.05 & 8.05 & 1.13 \\
\hline $496-498.5$ & 465.25 & 136.24 & $\mathrm{~F}$ & DND & 9.01 & 9.01 & 1.95 \\
\hline $498-500.5$ & 467.25 & 137.06 & M & DND & 9.61 & 9.61 & 2.46 \\
\hline $500-501$ & 468.5 & 137.60 & $\mathrm{~F}$ & 5 & 6.53 & 6.53 & -0.17 \\
\hline $500.5-503$ & 469.75 & 138.11 & $\mathrm{~F}$ & DND & 5.67 & 5.67 & -0.90 \\
\hline $507-509$ & 476 & 140.71 & $\mathrm{~F}$ & DND & 8.26 & 8.26 & 1.31 \\
\hline $509-513$ & 479 & 141.96 & M & DND & 9.52 & 9.52 & 2.38 \\
\hline $523-524$ & 491.5 & 147.15 & $\mathrm{~F}$ & 6 & 8.14 & 8.73 & 1.70 \\
\hline $523-524$ & 491.5 & 147.15 & $\mathrm{~F}$ & 6 & 9.31 & & \\
\hline $549-550$ & 517.5 & 157.96 & M & 6 & 8.17 & 8.17 & 1.23 \\
\hline $568-569$ & 536.5 & 168.34 & $\mathrm{~F}$ & 5 & 8.00 & 8.00 & 1.08 \\
\hline $581-582$ & 549.5 & 175.70 & $\mathrm{~F}$ & 3 & 8.77 & 8.67 & 1.65 \\
\hline $581-582$ & 549.5 & 175.70 & $\mathrm{~F}$ & 3 & 8.87 & & \\
\hline $581-582$ & 549.5 & 175.70 & $\mathrm{~F}$ & 3 & 8.38 & & \\
\hline $581-582$ & 549.5 & 175.70 & $\mathrm{~F}$ & 3 & 8.64 & & \\
\hline $620-621$ & 588.5 & 195.52 & $\mathrm{~F}$ & 4 & 9.69 & 9.69 & 2.53 \\
\hline $641-642$ & 609.5 & 204.37 & $\mathrm{~F}$ & 6 & 8.61 & 9.17 & 2.08 \\
\hline $641-642$ & 609.5 & 204.37 & $\mathrm{~F}$ & 5 & 10.00 & & \\
\hline $640-643$ & 609.5 & 204.37 & $\mathrm{~F}$ & 2 & 8.90 & & \\
\hline $660-661$ & 628.5 & 215.50 & M & 3 & 10.56 & 11.46 & 4.04 \\
\hline $660-661$ & 628.5 & 215.50 & $\mathrm{M}$ & 3 & 12.37 & & \\
\hline
\end{tabular}

${ }^{\text {a }}$ Corrected depth obtained by adding $32 \mathrm{~cm}$ to original depth due to break in core (see Boyle and Keigwin, 1985).

${ }^{\mathrm{b}}$ Uncorrected core depth (midpoint).

${ }^{c}$ VPI: visual preservation index; DND: did not determine.

${ }^{\mathrm{d}} \mathrm{Mean} \mathrm{Mg} / \mathrm{Ca}$ is the mean ratio for depth.

${ }^{\text {e }}$ BWT: estimated bottom water temperature.

values; all three indicators suggest a stronger AABW influence at these times. Conversely, during interglacial periods 5 and $1, \mathrm{Mg} / \mathrm{Ca}$ ratios were $11-12.5 \mathrm{mmol} / \mathrm{mol}$ and estimated BWTs were 3 to $>4{ }^{\circ} \mathrm{C}$. Higher $\mathrm{Mg} / \mathrm{Ca}$ ratios generally coincide with lower foraminiferal $\mathrm{Cd} / \mathrm{Ca}$ and higher values of $\delta^{13} \mathrm{C}$. During Terminations 1 and 2 the initial increase in $\mathrm{Mg} / \mathrm{Ca}$ appears to precede shifts in foraminiferal $\delta^{18} \mathrm{O}$ and $\delta^{13} \mathrm{C}$.
In order to examine the relationship between smoothed $\mathrm{Mg} / \mathrm{Ca}$ and orbitally driven changes in solar insolation, we plotted June insolation at $60^{\circ} \mathrm{N}$ in Fig. 2 on the right. There is a general correspondence between high insolation during the three substages of marine isotope stage 5, early stage 3 and early stage 1 , although it appears that insolation precedes the $\mathrm{Mg} / \mathrm{Ca}$ shift, especially during Terminations 1 and 2. 
Core CHN82-24-4PC (3427 m)

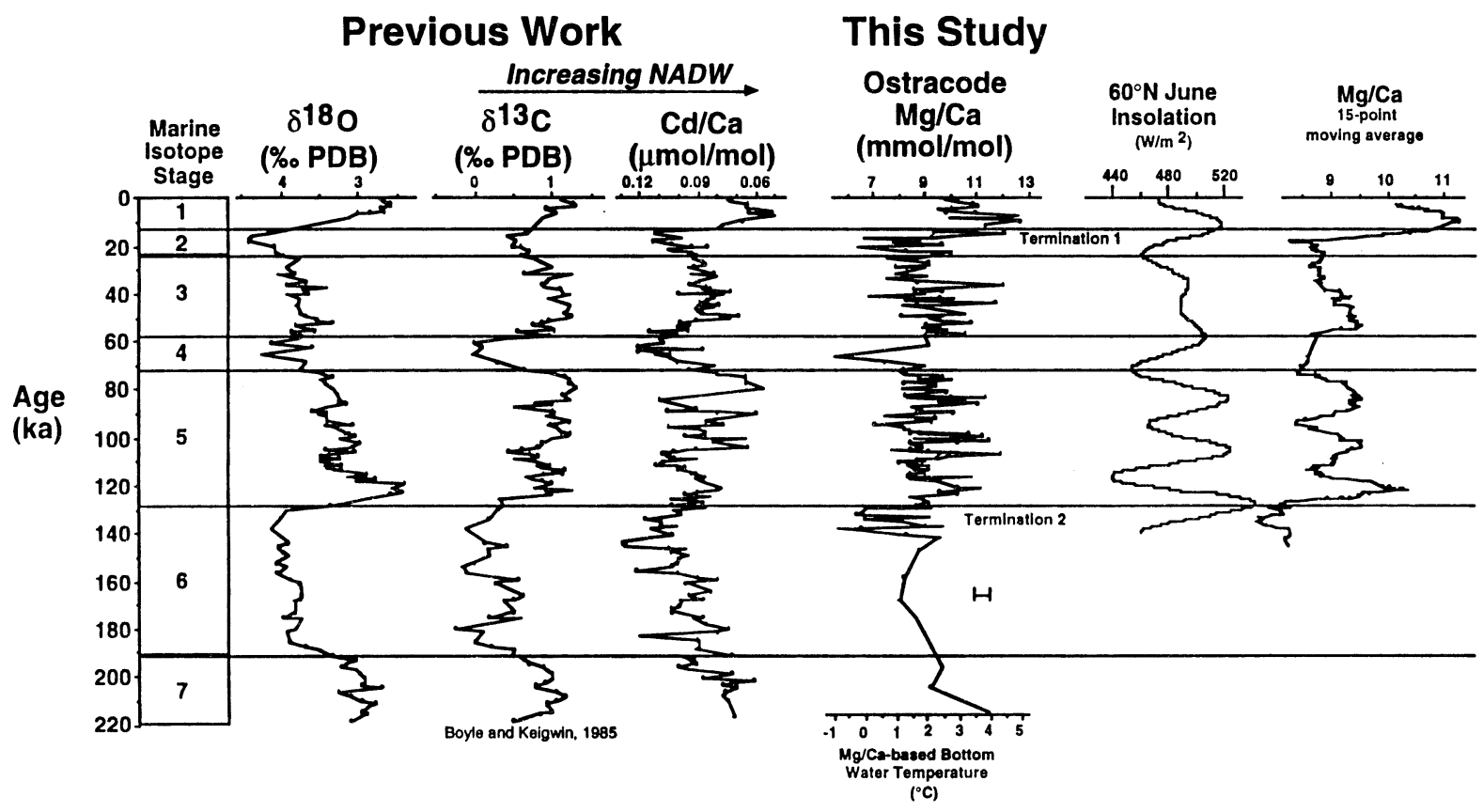

Fig. 2. Comparison of ostracod $\mathrm{Mg} / \mathrm{Ca}$ ratios with oxygen and carbon isotope and $\mathrm{Cd} / \mathrm{Ca}$ records from forams of Boyle and $\mathrm{Keigwin}$ (1985). At right, $\mathrm{Mg} / \mathrm{Ca}$ results are plotted as a 15-point moving average for comparison with $60^{\circ} \mathrm{N}$ solar insolation. See text.

There are several other noteworthy aspects of the long-term ostracod $\mathrm{Mg} / \mathrm{Ca}$ record shown in Fig. 2. First, $\mathrm{Mg} / \mathrm{Ca}$ ratios are higher (warmer BWTs) during marine isotope stage 1 compared with those for the last interglacial (LIG) maximum, stage 5e. This implies an extremely warm climate and strong NADW formation during the early Holocene. Second, $\mathrm{Mg} / \mathrm{Ca}$ ratios are lower during marine isotope stage 6 than during stage 2 (the last glacial maximum), apparently reflecting a more intense glacial period [see Crowley (1994)]. Third, there are apparent brief reversals in $\mathrm{Mg} / \mathrm{Ca}$ and BWT during Terminations 1 and 2 that perhaps correspond to short-term climatic events punctuating the last deglacial interval and the marine isotope stage 6-5 transition.

\subsection{Sub-orbital millennial scale variability}

There are also large-amplitude millennial-scale changes in Krithe $\mathrm{Mg} / \mathrm{Ca}$ ratios between about 110 and $10 \mathrm{ka}$, corresponding to the period encom- passing marine isotope stages 2 through $5 \mathrm{~d}$. In order to smooth potential variability due to sedimentary processes, intraspecific variability in $\mathrm{Mg} / \mathrm{Ca}$, and sub-millennial changes in BWT, Fig. 3 presents a three-point moving average of $\mathrm{Mg} / \mathrm{Ca}$ and estimated BWT compared with a record of the atmospheric temperature from the $\delta^{18} \mathrm{O}_{\text {ice }}$ in the Greenland GISP2 ice core. The GISP record provides evidence for relatively rapid millennialscale climate oscillations in which relatively light $\delta^{18} \mathrm{O}_{\text {ice }}$ values represent $\mathrm{D} / \mathrm{O}$ interstadial events. Fig. 3 also shows the age of Heinrich ice-rafting events which signify periods of ice-rafting deposition in the North Atlantic Ocean (Bond et al., 1992, 1997). This smoothing of the $\mathrm{Mg} / \mathrm{Ca}$ record yields a temporal resolution of slightly less than one $\mathrm{Mg} / \mathrm{Ca}$ measurement per 1000 years. Thus, it should be emphasized that the Chain core data are aliased by slow to moderate sedimentation rates and they do not capture all of the fine structure nor the extremes of temperature changes recorded in ice cores. 


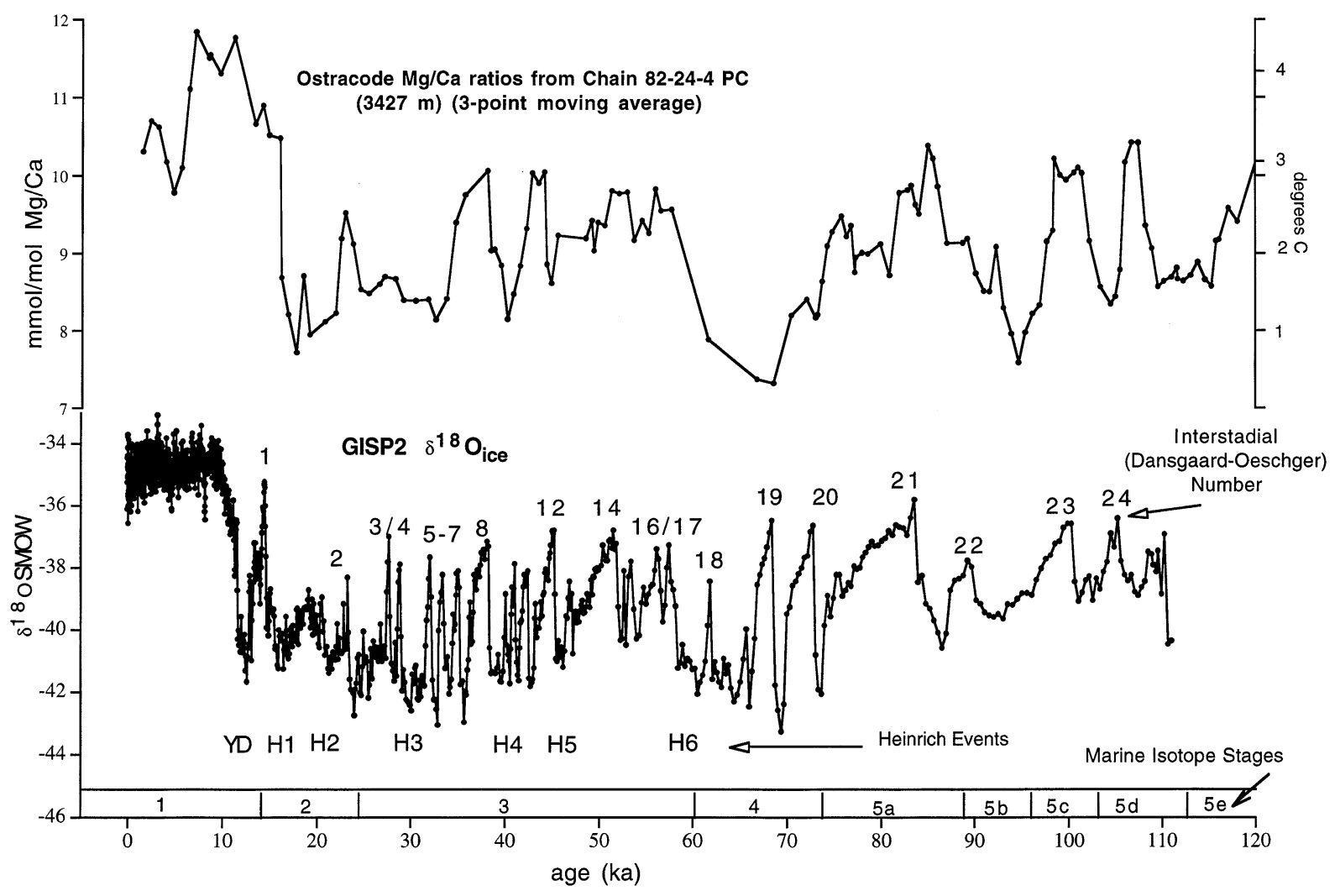

Fig. 3. Plot of three-point smoothed $\mathrm{Mg} / \mathrm{Ca}$ ratios of Chain core ostracods compared with GISP2 oxygen isotope record. D/O interstadial events \#1-\#24 are labeled. North Atlantic Ocean Heinrich Events (H1-H6) and the Younger Dryas (YD) are also labeled. Some $\mathrm{D} / \mathrm{O}$ events correspond to relatively high $\mathrm{Mg} / \mathrm{Ca}$ ratios (i.e. high BWT). See text.

Nonetheless, at least 14 interstadial warm BWT events between 110 and $10 \mathrm{ka}$ can be identified in the Chain core $\mathrm{Mg} / \mathrm{Ca}$ record (Fig. 3). The strongest of these are dated approximately at 32, 36-38, $43,48,73,85$ and $93 \mathrm{ka}$. Some of these may be correlated with $\mathrm{D} / \mathrm{O}$ interstadials. For example, high BWTs characterized this site during $\mathrm{D} / \mathrm{O}$ interstadials \#24, \#23, and \#21. The BWT at this site was about 2.5 to $3^{\circ} \mathrm{C}$, equal to that of the late Holocene, but about $1{ }^{\circ} \mathrm{C}$ cooler than the peak BWT reached during the Preboreal-early Holocene. The distinctive double peak in $\mathrm{Mg} / \mathrm{Ca}$ comprising D/O events \#24 and \#23 (the equivalent of marine isotope stage $5 \mathrm{c}$ ) is also recognized in carbonate and isotopic records from the BlakeBahama Outer Ridge (Keigwin et al., 1994). Although the correlation between the Chain core and GISP ice core isotope record is more tenuous,
$\mathrm{D} / \mathrm{O}$ interstadials \#12 and \#8 also seem to have been characterized by a relatively high deep North Atlantic BWT. Lesser peaks in $\mathrm{Mg} / \mathrm{Ca}$ ratios when the BWT at this site was about $2-2.5^{\circ} \mathrm{C}$ may correspond to other $\mathrm{D} / \mathrm{O}$ events, but this requires further confirmation in high sedimentation cores.

In comparing the deep North Atlantic $\mathrm{Mg} / \mathrm{Ca}$ record with the record of Heinrich events (Bond et al., 1992), we observe steep BWT declines for relatively strong Heinrich events (i.e. H-6 $\sim 69 \mathrm{ka}$, H-4 $\sim 37 \mathrm{ka}, \mathrm{H}-3 \sim 23 \mathrm{ka}$ and H-2 $\sim 16.5 \mathrm{ka}$ ) but little or no cooling occurred during H-5, $~ 52 \mathrm{ka}$.

\section{Discussion}

For some time, the idea that deep-sea BWTs declined during glacial periods has been supported 
by several paleoceanographic records (Emiliani, 1955; Chappell and Shackleton, 1986; Labeyrie et al., 1987). Our results provide compelling evidence for a glacial BWT in the North Atlantic of about $0.5^{\circ} \mathrm{C}$ during the coldest periods. This is about $2^{\circ} \mathrm{C}$ colder than temperatures in the region today and as much as $4^{\circ} \mathrm{C}$ below the maximum temperature reached during the early Holocene. The evidence also suggests that deep-sea temperatures during isotope stage 6 , the penultimate glaciation, were colder than temperatures during isotope stage 2 , the last glacial maximum. This is in line with terrestrial (glacial geology and lake levels) and marine (sea-surface temperatures) evidence reviewed by Crowley (1994) indicating that the penultimate glaciation was a more intense glacial period.

Moreover, the overall shape of the smoothed $\mathrm{Mg} / \mathrm{Ca}$ curve in Fig. 3 supports the hypothesis that orbital forcing exerts a first-order control on deep-sea circulation and BWTs, as discussed in detail by Imbrie et al. (1992). The timing and amplitude of the $\mathrm{Mg} / \mathrm{Ca}$ curve for the last $130 \mathrm{ka}$ resembles the characteristic shape of the June insolation curve and paleoclimate proxies such as ice volume. However, BWT changes appear to lag slightly behind solar insolation and lead benthic foraminiferal $\delta^{18} \mathrm{O} \quad(\sim$ ice volume $)$ and $\delta^{13} \mathrm{C}$ $(\sim \mathrm{AABW})$ during Terminations 1 and 2 . This relationship was also observed by Dwyer et al. (1995) for late Pliocene 41 ka obliquity cycles of $\mathrm{Mg} / \mathrm{Ca}$ at DSDP site 607, which is located in the same region as the Chain 82-24 core. Corrége and De Deckker (1997) also found a relationship between ostracod $\mathrm{Mg} / \mathrm{Ca}$ and summer insolation at $60^{\circ} \mathrm{S}$ between 25 and $75 \mathrm{ka}$ in the western Coral Sea.

Another noteworthy result is that the BWT in this part of the North Atlantic Ocean during the early Holocene (oxygen isotope stage 1) is warmer by as much as $1^{\circ} \mathrm{C}$ than temperatures during the LIG (isotope stage 5e). This difference may seem anomalous given that the sea level during the LIG was $\sim 5-6 \mathrm{~m}$ higher than present and there has been a consensus that the LIG was slightly warmer, at least in many regions, than the Holocene interglacial. One possible explanation is that early Holocene thermohaline circulation was more vig- orous than previously believed, leading to extremely warm deep-sea temperatures at the Chain core site.

Over millennial time scales, our evidence supports the hypothesis of cooling of deep-ocean waters during glacial periods and also indicates that North Atlantic deep BWTs also varied during the past $110 \mathrm{ka}$. These results support previous studies that have found evidence for millennialscale variability in other deep-sea and mid-depth proxies, including percent calcium carbonate (Keigwin and Jones, 1994), foraminiferal stable isotopes (Slowey and Curry, 1992; Keigwin et al., 1994; Oppo and Lehman, 1995; Lund and Mix, 1998) and foraminiferal $\mathrm{Cd} / \mathrm{Ca}$ ratios (Marchitto et al., 1998). Although precise correlations to the sequence of events that occur during a Heinrich event are not yet possible, the observed decreased deep-sea temperatures would be expected in the North Atlantic Ocean if the strength of thermohaline circulation diminished and warm NADW was reduced during the cooling phase of a Heinrich event (Keigwin and Lehman, 1994; Paillard and Labeyrie, 1994). We estimate that this deep-sea BWT variability between 110 and $20 \mathrm{ka}$ was about $1 / 2$ to $2 / 3$ the magnitude of that occurring during a complete glacial-interglacial cycle. The warmest BWTs attained during some interstadials during oxygen isotope stage 3 were $\sim 2.5$ to $3.0^{\circ} \mathrm{C}$, signifying strong NADW formation. Corrége and De Deckker (1997) also found evidence for BWTs in the Coral Sea during oxygen isotope stage 3 that were similar to present-day temperatures.

The distinct possibility exists that observed deep-sea temperature maxima during isotope stages $2-4$ correspond to $\mathrm{D} / \mathrm{O}$ interstadial events recorded in Greenland ice cores. However, we urge caution in attempting to correlate individual $\mathrm{D} / \mathrm{O}$ events with sediment core $\mathrm{Mg} / \mathrm{Ca}$ records until analyses from cores taken in regions with high sedimentation rate and sensitive to changes in NADW and AABW variability can be carried out. Still, our initial results suggest that millennial-scale oscillations of climate in the North Atlantic may be capable of affecting global climate by way of changes in thermohaline circulation manifested by deep bottom water changes. Strong NADW formation would lead to large ocean-to-atmosphere 
heat flux in high latitudes, an idea that is qualitatively consistent with the magnitude of atmospheric warming of $5-8^{\circ} \mathrm{C}$ over Greenland during D/O cycles (GRIP, 1993; Mayewski et al., 1996). Our observations are also consistent with ocean modeling studies by Rahmstorf (1994) that demonstrate a decrease in thermohaline circulation and lower BWT during glacial periods.

\section{Acknowledgements}

This work was supported by USGS Climate Program and NSF Grant OPP-09400250. We thank E. Boyle and L. Keigwin for Chain core samples, W. Curry and J. Broda for coretops samples, P. De Deckker for comments on ostracod shell calcification, E. Klein for access to Duke Geology Department DCP. T. Corrége, H. Dowsett, J. Holmes, S. Verardo, and S. Ishman provided very useful reviews.

\section{References}

Behl, R.J., Kennett, J.P., 1996. Brief interstadial events in the Santa Barbara basin, NE Pacific, during the past $60 \mathrm{kyr}$. Nature 379, 243-246.

Benson, L.V., Burdett, J.W., Kashgarian, M., Lund, S.P., Phillips, F.M., Rye, R.O., 1996. Climatic and hydrologic oscillations in the Owens Lake Basin and adjacent Sierra Nevada, California. Science 274, 746-749.

Bond, G.C., Lotti, R., 1995. Iceberg discharges into the North Atlantic on millennial time scales during the last glaciation. Science 267, 1005-1010

Bond, G., Heinrich, H., Broecker, W., Labeyrie, L., McManus, J., Andrews, J., Huon, S., Jantschik, R., Clasen, S., Simet, C., Tedesco, K., Klas, M., Bonani, G., Ivy, S., 1992. Evidence for massive discharges of icebergs into the North Atlantic ocean during the last glacial period. Nature 360, 245-249.

Bond, G., Showers, W., Cheseby, M., Lotti, R., Almasi, P., deMenocal, P., Priore, P., Cullen, H., Hajdas, I., Bonani, G., 1997. A pervasive millennial-scale cycle in North Atlantic Holocene and glacial climates. Science 278, 1257-1266.

Boyle, E.A., Keigwin, L.D., 1985. Comparison of Atlantic and Pacific paleochemical records for the last 215,000 years: changes in deep ocean circulation and chemical inventories. Earth Planet. Sci. Lett 76, 135-150

Broecker, W.S., 1994. Massive iceberg discharges as triggers for global climate change. Nature 372, 421-424.

Cadot Jr, H.M., Kaesler, R.L., 1977. Magnesium content of calcite in carapaces of benthic marine ostracods. Univ. Kansas Paleont. Contrib. Pap. 87, 1-23.

Chappell, J., Shackleton, N.J., 1986. Oxygen isotopes and sea level. Nature 324, 137-140.

Coles, G., Whatley, R.C., Moguilevsky, A., 1994. The ostracod genus Krithe from the Tertiary and Quaternary of the North Atlantic. Palaeontology 37, 71-120.

Corrége, T., 1993. Preliminary results of paleotemperature reconstruction using the magnesium to calcium ratio of deep-sea ostracod shells from the Late Quaternary of Site 822, Leg 133 (western Coral Sea). Proc. ODP Sci. Results $133,175-180$.

Corrége, T., De Deckker, P., 1997. Faunal and geochemical evidence for changes in intermediate water temperature and salinity in the western Coral Sea (northeast Australia) during the late Quaternary. Palaeogeogr. Palaeoclimatol. Palaeoecol. 313, 183-205.

Cronin, T.M., Dwyer, G.S., Baker, P.A., Rodriguez-Lazaro, J., Briggs Jr, W.A., 1996. Deep-sea ostracod shell chemistry (Mg:Ca ratios) and late Quaternary Arctic Ocean history. In: Andrews, J.T., Austin, W.E.N., Bergsten, H., Jennings, A. (Eds.), Late Quaternary Paleoceanography of the North Atlantic Margins. In: Geological Society (London) Special Publication no. 111, 117-134.

Cronin, T.M., DeMartino, D.M., Dwyer, G.S., RodriguezLazaro, J., 1999. Deep-sea ostracode species diversity: response to late Quaternary climate change. Mar. Micropal. 37, 231-249.

Crowley, T.J., 1994. Potential reconciliation of Devils Hole and deep-sea Pleistocene chronologies. Paleoceanography 9, $1-5$.

De Deckker, P., Chivas, A.R., Shelley, J.M.G., 1999. Uptake of $\mathrm{Mg}$ and $\mathrm{Sr}$ in the euryhaline ostracod Cyprideis determined from in vitro experiments. Palaeogeogr. Palaeoclimatol. Palaeoecol. 148, 105-116.

Dwyer, G.S., Cronin, T.M., Baker, P.A., Raymo, M.E., Buzas, J.S., Corrége, T., 1995. North Atlantic deep-water temperature change during late Pliocene and late Quaternary climatic cycles. Science 270, 1147-1151.

Dwyer, G.S., Cronin, T.M., Baker, P.A., Rodriguez-Lazaro, J., 2000. Changes in North Atlantic deep-sea temperature since the last glacial maximum. Geochem., Geophys., Geosys., In preparation.

Emiliani, C., 1955. Pleistocene temperatures. J. Geol. 63, $538-578$.

Greenland Ice-Core Project (GRIP) Members, 1993. Climate instability during the last interglacial period recorded in the GRIP ice core. Nature 364, 203-207.

Grimm, E.C., Jacobson, G.L., Watts, W.A., Hansen, B.C.S., Maasch, K.A., 1993. A 50,000-year record of climate oscillations from Florida and its temporal correlation with Heinrich events. Science 261, 198-200.

Imbrie, J.A., Boyle, E.A., Clemens, S.C., Duffy, A., Howard, W.R., Kukla, G., Kutzbach, J., Martinson, D.G., McIntyre, A., Mix, A.C., Molfino, B., Morley, J.J., Peterson, L.C., Pisias, N.G., Prell, W.L., Raymo, M.E., Shackleton, N.J., Toggweiler, J.R., 1992. On the structure and origin of major 
glaciation cycles, 1, Linear responses to Milankovitch forcing. Paleoceanography 7, 701-738.

Johnsen, S.J., Clausen, H.B., Dansgaard, W., Fuhrer, K., Gundestrup, N., Hammer, C.U., Iversen, P., Jouzel, J., Stauffer, B., Steffensen, J.P., 1992. Irregular glacial interstadials recorded in a new Greenland ice core. Nature 359, 311-313.

Keigwin, L.D., Jones, G.A., 1994. Western North Atlantic evidence for millennial-scale changes in ocean circulation and climate. J. Geophys. Res. 99, C6, 12,397-12,410.

Keigwin, L.D., Lehman, S.J., 1994. Deep circulation change linked to Heinrich event 1 and Younger Dryas in a middepth North Atlantic core. Paleoceanography 9, 185-194.

Keigwin, L.D., Curry, W.B., Lehman, S.J., Johnsen, S., 1994. The role of the deep ocean in North Atlantic climate change between 70 and $130 \mathrm{kyr}$ ago. Nature 371, 323-326.

Labeyrie, L.D., Duplessy, J.C., Blanc, P.L., 1987. Variations in mode of formation and temperature of oceanic deep waters over the past 125,000 years. Nature 327, 477-482.

Lund, D.C., Mix, A.C., 1998. Millennial-scale deep water oscillations: Reflections of the North Atlantic in the deep Pacific from 10 to $60 \mathrm{ka}$. Paleoceanography 13, 10-19.

Majoran, S., Agrenius, S., Dwyer, G.S., 2000. The effect of temperature on the geochemical composition of the valves of the ostracod species Krithe praetexta praetexta. In: Nazik, A. (Ed.), Studies on Ostracoda, Proceedings of the 4th European Ostracodologists Meeting, Adana, 5-8 July, 1999.

Marchitto Jr, T.M., Curry, W.B., Oppo, D.W., 1998. Millennial-scale changes in North Atlantic circulation since the last glaciation. Nature 393, 557-561.

Mayewski, P.A., Twickler, M.S., Whitlow, S.I., Meeker, L.D., Yang, Q., Thomas, J., Kreutz, K., Grootes, P.M., Morse,
D.L., Steig, E.J., Waddington, E.D., Saltzman, E.S., Whung, P.-Y., Taylor, K.C., 1996. Climate change during the last deglaciation in Antarctica. Science 272, 1636-1638.

Muller, A., Nürnberg, D., 1997. Tracing the Mediterranean outflow water by $\mathrm{Mg} / \mathrm{Ca}$ and $\mathrm{Sr} / \mathrm{Ca}$ ratios in benthic ostracods. EOS AGU Trans. 78, suppl., F360.

Oppo, D.W., Lehman, S.J., 1995. Suborbital timescale variability of North Atlantic deep water during the past 200,000 years. Paleoceanography 10, 901-910.

Paillard, D., Labeyrie, L., 1994. Role of thermohaline circulation in the abrupt warming after Heinrich events. Nature $372,162-164$.

Raymo, M.E., Hodell, D., Jansen, E., 1992. Response of deep ocean circulation to initiation of Northern Hemisphere glaciation (3-2 Ma). Paleoceanography 7, 645-672.

Raymo, M.E., Ganley, K., Carter, S., Oppo, D.W., McManus, J.F., 1998. Millennial-scale climate instability during the early Pleistocene epoch. Nature 392, 699-702.

Rahmstorf, S., 1994. Rapid climate transitions in a coupled ocean-atmosphere model. Nature 372, 82-85.

Slowey, N.C., Curry, W.B., 1992. Enhanced ventilation of the North Atlantic subtropical gyre thermocline during the last glaciation. Nature 358, 665-668.

Steig, E.J., Brook, E.J., White, J.W.C., Sucher, C.M., Bender, M.L., Lehman, S.J., Morse, D.L., Waddington, E.D., Clow, G.D., 1998. Synchronous climate changes in Antarctica and the North Atlantic. Science 282, 92-95.

Taylor, K.C., Lamorey, G.W., Doyle, G.A., Alley, R.B., Grootes, P.M., Mayewski, P.A., White, J.W.C., Barlow, L.K., 1993. The "flickering switch" of late Pleistocene climate change. Nature 361, 432-436. 